\title{
OPEN Risk factors and evaluation of keratoconus progression after penetrating keratoplasty with anterior segment optical coherence tomography
}

\begin{abstract}
Junko Yoshida ${ }^{1,2 \bowtie}$, Tetsuya Toyono ${ }^{1}$, Rika Shirakawa ${ }^{1}$, Takashi Miyai ${ }^{1}$ \& Tomohiko Usui ${ }^{1,2}$
To determine the risk factors and unique characteristics of keratoconus $(\mathrm{KC})$ progression after penetrating keratoplasty (PK), anterior segment optical coherence tomography parameters were statistically analyzed in comparison with eyes undergoing PK for other diseases as a control. Ninetyone eyes maintaining clear PK grafts for over 10 years were divided into 2 groups according to the primary indication for PK (KC vs Others groups). Corneal thinning indicators (inferior host thinnest corneal thickness/central corneal thickness [IHT/CCT], inferior graft thinnest corneal thickness/CCT [IGT/CCT]), were smaller whereas anterior chamber depth, and steepest corneal power (Ks), and maximum corneal power $\left(\mathrm{K}_{\max }\right)$ were larger in the $\mathrm{KC}$ group with statistical significance. Graft size, $\mathrm{K}_{\max }$ and Ks correlated with IHT/CCT and IGT/CCT in the KC group. These correlations were not detected in controls. Graft size and postoperative period were selected by multivariate regression analysis as factors for corneal ectatic changes in the KC group. In conclusion, KC eyes long after PK show inferior graft and host corneal thinning, and corneal protrusion. Corneal power parameters such as $\mathrm{K}_{\max }$ or $\mathrm{Ks}$ can be used to monitor KC progression after PK. A small graft might lead to KC progression after PK.
\end{abstract}

Keratoconus (KC) is a bilateral, progressive, and non-inflammatory corneal ectatic disease whose clinical characteristics are paracentral corneal thinning and corneal protrusion, mainly occurring in juveniles ${ }^{1,2}$. The KC prevalence in the latest report was higher (1:375) than expected based on previous reports, probably a result of advances in imaging techniques ${ }^{3}$. According to the US Eye Bank statistical report in 2017, 38,000 penetrating keratoplasties (PK) are performed annually, primarily for $\mathrm{KC}$, which accounts for $\sim 25 \%$ of the indication-known PK cases. The prognosis of PK for KC eyes is excellent, with an over $90 \% 10$-year graft survival rate ${ }^{4-6}$. KC sometimes recurs in the long-term-usually decades-after PK. As there are few reports ${ }^{7-10}$ on recurrent KC, the details of recurrence, such as the frequency and characteristics, remain unknown.

We recently reported that KC recurs in up to $36 \%$ of $\mathrm{PK}$ patients who had maintained clear grafts for over 10 years $^{8}$. Also, in a report of acute hydrops cases in recurrent $\mathrm{KC}^{7}$, Descemet's membrane breaks are seen around the graft-host junction, not around the center as usually seen in keratoplasty-naive KC. The characteristics are unsurprisingly different between recurrent and keratoplasty-naïve KCs. Recurrent KC might thus not be a rare condition in long-surviving grafts. In addition, as the efficacy of corneal collagen crosslinking to prevent KC progression was recently recognized worldwide ${ }^{11-17}$, corneal collagen crosslinking might need to be considered for recurrent $\mathrm{KCs}^{18}$. In that case, proper evaluation of $\mathrm{KC}$ progression after $\mathrm{PK}$ is necessary, and it is important to clarify the characteristics and diagnostic parameters. The problem here, however, is that there are no clear and objective diagnosing criteria of recurrent $\mathrm{KC}$ and thus, whole aspect of $\mathrm{KC}$ progression after $\mathrm{PK}$ is still unclear.

The present study aimed to determine the objective characteristics of KC progression after PK, such as corneal thinning or ectatic changes, using anterior segment optical coherence tomography (AS-OCT), in comparison with eyes undergoing PK eyes for other diseases as a control. We also explored the risk factors for KC progression after PK. A retrospective comparative analysis of post-PK eyes with a greater than 10-year follow-up period was performed.

\footnotetext{
${ }^{1}$ Department of Ophthalmology, The University of Tokyo Hospital, 7-3-1 Hongo, Bunkyo-ku, Tokyo 113-8655, Japan. ${ }^{2}$ Department of Ophthalmology, The International University of Health and Welfare, 1-4-3, Mita, Minato-ku, Tokyo 108-8329, Japan. ${ }^{\varpi}$ email: junkoyoshida-tky@umin.ac.jp
} 


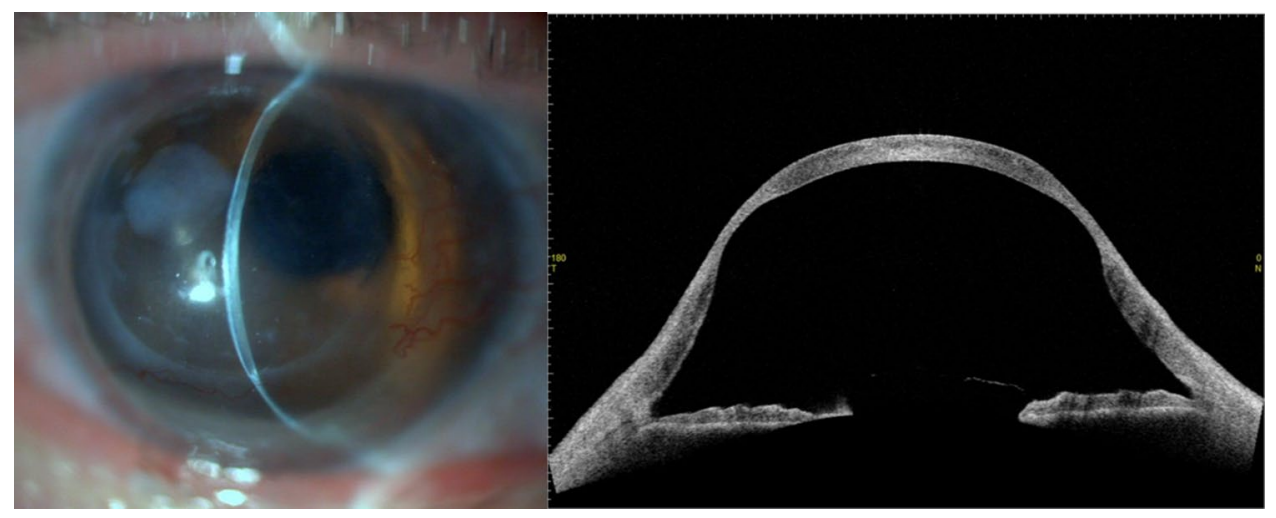

Figure 1. Representative images of an eye of 65-year-old man who underwent penetrating keratoplasty for keratoconus 36 years ago. The corneal thinning in the mid-peripheral area and corneal protrusion are remarkable.

\section{Results}

Representative images of $\mathrm{KC}$ recurrence are shown in Fig. 1. The patient characteristics and parameters measured by AS-OCT are shown in Table 1. The KC group comprised 46 eyes and the Others group comprised 45 eyes. Postoperative periods (approximately 25 years) did not differ between the 2 groups. Mean graft size was $7.0 \mathrm{~mm}$ in the KC group and $7.3 \mathrm{~mm}$ in Others group $(p=0.042)$. Anterior chamber depth (ACD) was $3.67 \mathrm{~mm}$ in $\mathrm{KC}$ group and $2.95 \mathrm{~mm}$ in Others group $(p<0.0001)$. The inferior corneal thinning indicators (inferior host thinnest corneal thickness/central corneal thickness [IHT/CCT] and inferior graft thinnest corneal thickness/CCT [IGT/ CCT]) were smaller in the KC group ( $p=0.00034$ and $p=0.025$, respectively). Keratometric values, such as the steepest corneal power (Ks), average corneal power $(\mathrm{AvgK})$ and maximum keratometric corneal power $\left(\mathrm{K}_{\max }\right)$, were significantly larger in the KC group.

Figure 2 shows the distributions of the corneal ectasia indicators (IHT/CCT, IGT/CCT, and ACD) in each group. IHT/CCT peaked at around 0.6-0.7 in the KC group and at 1.1-1.2 in the Others group. IGT/CCT peaked at $0.9-1.0$ in the KC group and at 1.0-1.1 in the Others group.

Correlation coefficients in each group are shown in Table 2. In the KC group, graft size was strongly correlated with the corneal thinning indicators (IHT/CCT and IGT/CCT) and keratometric parameters, but this tendency was not observed in the Others group. IHT/CCT and IGT/CCT were correlated with most of the keratometric parameters in the KC group. In the Others group, IHT/CCT and IGT/CCT were correlated with age, but this was not observed in the $\mathrm{KC}$ group. Figure 3 shows scatterplots between $\mathrm{K}_{\max }$ and the corneal ectasia indicators. A negative correlation was detected between $\mathrm{K}_{\max }$ and IHT/CCT or IGT/CCT in the KC group, but not in the Others group. Both groups showed a tendency toward a positive correlation between $\mathrm{K}_{\max }$ and ACD.

Table 3 shows the results of the multivariant regression analysis with the corneal ectasia indicators as dependent variables and the patient background characteristics as independent variables, aiming to determine the risk factors for corneal ectatic changes. Graft size was selected as explaining the corneal thinning indicators with negative estimates in the KC group, but not in the Others group. Graft size was also selected as explaining ACD in both groups, negatively in the KC group and positively in the Others group. Table 4 shows the results of the multivariant regression analysis to determine which corneal ectasia indicators were associated with corneal powers and astigmatisms. In the KC group, IHT/CCT was selected as explaining all of the keratometric parameters, but this was not the case in the Others group.

\section{Discussion}

In this study, we performed a comparative analysis of patients who underwent $\mathrm{PK}$ for $\mathrm{KC}$ and other diseases as a control to determine the characteristics and risk factors of KC progression after PK using AS-OCT data. The mean postoperative period was over 25 years, which is sufficiently long to determine the corneal ectatic change. To quantify corneal thinning, we calculated corneal thinning indicators: IHT/CCT and IGT/CCT, each of which explains the relative thickness in the inferior host cornea and inferior graft cornea, respectively. Also, we used $\mathrm{ACD}$ as a corneal protrusion parameter. These three parameters were set to evaluate corneal ectasia in this study.

Comparing the KC and Others groups, KC had a deeper ACD, and smaller IHT/CCT and IGT/CCT. The normal cornea is thinnest at the center and gradually becomes thicker nearer the peripheral area. An IHT/CCT or IGT/CCT of less than or nearly 1.0 indicates inferior corneal thinning. The peak IHT/CCT was around 0.6-0.7 in the KC group and around 1.1-1.2 in the Others group (Fig. 2). Likewise, the normal ACD should be $\sim 3.0 \mathrm{~mm}$, which was indeed observed in the Others group, but the peak of the KC group fell to around 3.5-4.0, suggesting corneal protrusion in KC eyes. Those results suggest KC eyes showed a corneal ectatic change. This corneal ectatic change is also supported by the larger values of keratometric parameters such as Ks, AvgK, Sph, and $\mathrm{K}_{\max }$. In contrast, astigmatic parameters such as cylinder (CYL), regular astigmatism (Reg), asymmetry (Asym), or higher order irregularity (HOI) did not differ between the groups, probably because of the nature of PK, which causes strong astigmatism. Thus, in post-PK eyes, astigmatic change should not be important for evaluating KC progression, although astigmatism is usually important for judging progression in keratoplasty-naïve KC eyes. 


\begin{tabular}{|c|c|c|c|c|c|}
\hline & \multicolumn{2}{|c|}{ KC $(\mathrm{N}=46)$} & \multicolumn{2}{|c|}{ Others $(\mathrm{N}=45)$} & \multirow{2}{*}{$p$} \\
\hline & Mean & SD & Mean & SD & \\
\hline Age at PK (years) & 29.5 & 9.4 & 46.1 & 18.1 & $<0.0001$ \\
\hline Postoperative period (years) & 27.7 & 8.6 & 25.5 & 10.5 & 0.27 \\
\hline Current age (years) & 57.2 & 10.3 & 71.6 & 15.5 & $<0.0001$ \\
\hline Man to woman ratio (\%) & 76.1 & - & 44.4 & - & 0.0027 \\
\hline Graft size $(\mathrm{mm})$ & 7.0 & 0.69 & 7.3 & 0.61 & 0.042 \\
\hline $\mathrm{ACD}(\mathrm{mm})$ & 3.67 & 0.72 & 2.95 & 0.63 & $<0.0001$ \\
\hline CCT $(\mu \mathrm{m})$ & 570 & 58.1 & 562 & 68.0 & 0.52 \\
\hline $\operatorname{IHT}(\mu \mathrm{m})$ & 509 & 165.7 & 624 & 119.0 & 0.00027 \\
\hline IGT $(\mu \mathrm{m})$ & 585 & 91.0 & 618 & 96.3 & 0.093 \\
\hline IHT/CCT & 0.90 & 0.32 & 1.12 & 0.23 & 0.00034 \\
\hline IGT/CCT & 1.03 & 0.16 & 1.11 & 0.17 & 0.025 \\
\hline Ks (D) & 57.5 & 8.8 & 51.5 & 6.5 & 0.00035 \\
\hline $\operatorname{AvgK}(\mathrm{D})$ & 53.2 & 7.5 & 48.2 & 5.9 & 0.00061 \\
\hline CYL (D) & 8.5 & 6.3 & 6.5 & 4.3 & 0.077 \\
\hline \multicolumn{6}{|l|}{ Fourier analysis $(D)$} \\
\hline Spherical & 52.9 & 7.4 & 48.1 & 5.9 & 0.00089 \\
\hline Regular astigmatism & 4.4 & 3.1 & 3.4 & 2.2 & 0.083 \\
\hline Asymmetry & 4.0 & 4.3 & 3.4 & 2.5 & 0.38 \\
\hline HOI & 0.85 & 0.50 & 1.02 & 1.03 & 0.32 \\
\hline $\mathrm{K}_{\max }(\mathrm{D})$ & 66.9 & 14.5 & 59.5 & 11.0 & 0.012 \\
\hline
\end{tabular}

Table 1. Patient characteristics and measured parameters in the $\mathrm{KC}$ and Others groups. $K C$ keratoconus, $P K$ penetrating keratoplasty, $A C D$ anterior chamber depth, CCT central corneal thickness, IHT inferior thinnest host cornea thickness, IGT inferior thinnest graft thickness, IHT/CCT IHT ratio to CCT, IGT/CCT IGT ratio to CCT, Ks steepest corneal power, D diopters, $A v g K$ average corneal power, $C Y L$ cylinder, $H O I$ higher order irregularity, $K_{\max }$ Maximum corneal power.

IHT/CCT

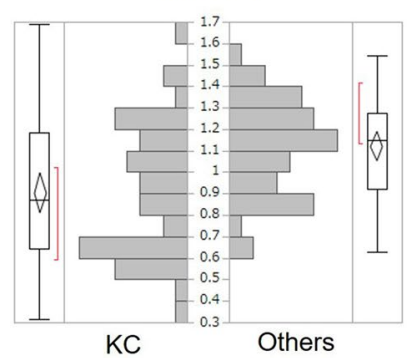

IGT/CCT

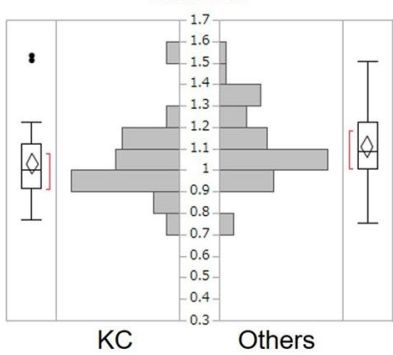

ACD

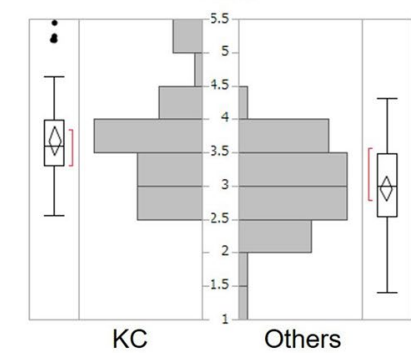

Figure 2. Distributions of corneal ectasia indicators (IHT/CCT, IGT/CCT, and ACD) in the KC and Others groups. Both the inferior host cornea and graft were thinner in the $\mathrm{KC}$ group, but the tendency was more obvious in the host cornea. The ACD tend to be larger in the KC group, suggesting more severe corneal protrusion.

Pearson correlation coefficients revealed interesting differences between the KC and Others groups. First, positive correlations between the graft size and corneal thinning parameters observed in the KC group were not observed in the Others group. Graft size was also negatively correlated with keratometric parameters in the $\mathrm{KC}$ group, but not in the Others group. In other words, a smaller graft may cause more thinning and more severe protrusion, i.e., KC progression. Second, negative correlations between corneal thinning parameters and keratometric parameters were observed in the $\mathrm{KC}$ group, but not in the Others group. This suggests that inferior corneal thinning is related to stronger keratometric values, and thus those parameters are reliable for evaluating KC progression after PK. Positive correlations between ACD and keratometic parameters were common to both groups, indicating that eyes with a deeper ACD tend to have a stronger corneal power regardless of the primary indications for PK. This supports the rationale for using ACD as a corneal protrusion parameter, although ACD sometimes depends on cataract formation.

As described above, the corneal ectasia parameters that we set (IHT/CCT, IGT/CCT, and ACD) can probably be used as indicators of KC progression after PK. Next, we performed multivariant regression analysis to determine the risk factors for KC progression. From the examined candidate risk factors (age at PK, current age, 


\begin{tabular}{|c|c|c|c|c|c|c|c|c|c|c|c|c|c|c|}
\hline & \multirow[b]{2}{*}{ Age at PK } & \multirow{2}{*}{$\begin{array}{l}\text { Postop } \\
\text { period }\end{array}$} & \multirow[b]{2}{*}{ Graft size } & \multirow[b]{2}{*}{$\mathrm{ACD}$} & \multirow[b]{2}{*}{ IHT/CCT } & \multirow[b]{2}{*}{ IGT/CCT } & \multirow[b]{2}{*}{ Ks } & \multirow[b]{2}{*}{ AvgK } & \multirow[b]{2}{*}{ CYL } & \multicolumn{4}{|c|}{ Fourier analysis } & \multirow[b]{2}{*}{$\mathbf{K}_{\max }$} \\
\hline & & & & & & & & & & Sph & Reg & Asym & HOI & \\
\hline \multicolumn{15}{|l|}{ KC group } \\
\hline Current age & $\underline{0.628}$ & $\underline{0.517}$ & -0.249 & 0.005 & -0.040 & 0.061 & 0.167 & 0.096 & 0.238 & 0.075 & 0.259 & 0.217 & $\underline{0.354}$ & 0.055 \\
\hline$p$ & $\leq 0.0001$ & $\underline{0.001}$ & 0.121 & 0.975 & 0.809 & 0.710 & 0.304 & 0.556 & 0.138 & 0.646 & 0.106 & 0.178 & $\underline{0.025}$ & 0.735 \\
\hline Age at PK & & $-\underline{0.342}$ & 0.081 & 0.108 & -0.113 & 0.026 & 0.214 & 0.147 & 0.251 & 0.132 & 0.291 & 0.227 & $\underline{0.402}$ & 0.157 \\
\hline$p$ & & $\underline{0.031}$ & 0.621 & 0.507 & 0.487 & 0.873 & 0.184 & 0.366 & 0.118 & 0.418 & 0.069 & 0.159 & $\underline{0.010}$ & 0.334 \\
\hline $\begin{array}{l}\text { Postop } \\
\text { period }\end{array}$ & & & $-\underline{0.389}$ & -0.120 & 0.077 & 0.044 & -0.035 & -0.046 & 0.012 & -0.054 & -0.007 & 0.013 & -0.015 & -0.103 \\
\hline $\mathrm{p}$ & & & $\underline{0.013}$ & 0.462 & 0.637 & 0.785 & 0.833 & 0.779 & 0.942 & 0.739 & 0.968 & 0.938 & 0.927 & 0.526 \\
\hline Graft size & & & & -0.265 & $\underline{0.503}$ & $\underline{0.564}$ & $-\underline{0.417}$ & $-\underline{0.439}$ & -0.124 & $-\underline{0.439}$ & -0.135 & $-\underline{0.339}$ & $-\underline{0.368}$ & $-\underline{0.434}$ \\
\hline$p$ & & & & 0.098 & $\underline{0.001}$ & $\leq 0.0001$ & $\underline{0.007}$ & $\underline{0.005}$ & 0.447 & $\underline{0.005}$ & 0.406 & $\underline{0.032}$ & $\underline{0.019}$ & $\underline{0.005}$ \\
\hline ACD & & & & & $-\underline{0.622}$ & $-\underline{0.438}$ & $\underline{0.543}$ & $\underline{0.603}$ & 0.073 & $\underline{0.608}$ & 0.120 & $\underline{0.368}$ & $\underline{0.359}$ & $\underline{0.668}$ \\
\hline$p$ & & & & & $\leq 0.0001$ & $\underline{0.005}$ & $\underline{0.0003}$ & $\leq 0.0001$ & 0.656 & $\leq 0.0001$ & 0.460 & $\underline{0.020}$ & $\underline{0.023}$ & $\leq 0.0001$ \\
\hline $\mathrm{IHT} / \mathrm{CCT}$ & & & & & & $\underline{0.754}$ & $-\underline{0.696}$ & $-\underline{0.658}$ & $-\underline{0.383}$ & $-\underline{0.645}$ & $-\underline{0.427}$ & $-\underline{0.576}$ & $-\underline{0.523}$ & $-\underline{0.759}$ \\
\hline$p$ & & & & & & $\leq 0.0001$ & $\leq 0.0001$ & $\leq 0.0001$ & $\underline{0.015}$ & $\leq 0.0001$ & $\underline{0.006}$ & $\underline{0.0001}$ & $\underline{0.001}$ & $\leq 0.0001$ \\
\hline IGT/CCT & & & & & & & $-\underline{0.530}$ & $-\underline{0.534}$ & -0.212 & $-\underline{0.531}$ & -0.222 & $-\underline{0.401}$ & $-\underline{0.327}$ & $-\underline{0.556}$ \\
\hline$p$ & & & & & & & $\underline{0.0004}$ & $\underline{0.0004}$ & 0.189 & $\underline{0.0004}$ & 0.168 & $\underline{0.010}$ & $\underline{0.040}$ & $\underline{0.0002}$ \\
\hline \multicolumn{15}{|c|}{ Others group } \\
\hline Current age & $\underline{0.815}$ & 0.069 & 0.044 & -0.059 & 0.275 & 0.183 & 0.093 & 0.112 & -0.029 & 0.085 & -0.101 & 0.200 & -0.037 & 0.028 \\
\hline$p$ & $\leq 0.0001$ & 0.674 & 0.786 & 0.718 & 0.086 & 0.259 & 0.570 & 0.490 & 0.861 & 0.604 & 0.535 & 0.216 & 0.819 & 0.862 \\
\hline Age at PK & & $-\underline{0.522}$ & 0.143 & 0.150 & $\underline{0.423}$ & $\underline{0.275}$ & 0.249 & 0.243 & 0.084 & 0.223 & 0.045 & 0.382 & 0.064 & 0.265 \\
\hline$P$ & & $\underline{0.001}$ & 0.377 & 0.354 & $\underline{0.006}$ & $\underline{0.086}$ & 0.121 & 0.130 & 0.605 & 0.167 & 0.784 & 0.015 & 0.695 & 0.099 \\
\hline $\begin{array}{l}\text { Postop } \\
\text { period }\end{array}$ & & & -0.182 & $-\underline{0.346}$ & $-\underline{0.325}$ & -0.205 & -0.293 & -0.253 & -0.187 & -0.259 & -0.226 & $-\underline{0.363}$ & -0.165 & $-\underline{0.424}$ \\
\hline$p$ & & & 0.261 & $\underline{0.029}$ & $\underline{0.041}$ & 0.205 & 0.067 & 0.115 & 0.248 & 0.106 & 0.162 & $\underline{0.021}$ & 0.308 & $\underline{0.006}$ \\
\hline Graft size & & & & $\underline{0.355}$ & 0.260 & -0.007 & 0.037 & 0.012 & 0.078 & 0.028 & 0.128 & -0.022 & 0.099 & 0.149 \\
\hline$p$ & & & & $\underline{0.025}$ & 0.106 & 0.965 & 0.820 & 0.941 & 0.632 & 0.863 & 0.430 & 0.895 & 0.543 & 0.359 \\
\hline $\mathrm{ACD}$ & & & & & 0.089 & -0.017 & $\underline{0.316}$ & $\underline{0.321}$ & 0.075 & $\underline{0.360}$ & 0.155 & 0.162 & $\underline{0.332}$ & $\underline{0.517}$ \\
\hline$p$ & & & & & 0.587 & 0.915 & $\underline{0.047}$ & $\underline{0.044}$ & 0.645 & $\underline{0.023}$ & 0.338 & 0.319 & $\underline{0.036}$ & $\underline{0.001}$ \\
\hline IHT/CCT & & & & & & $\underline{0.752}$ & -0.078 & -0.088 & 0.007 & -0.094 & 0.009 & 0.151 & -0.047 & -0.015 \\
\hline$p$ & & & & & & $\leq 0.0001$ & 0.633 & 0.587 & 0.965 & 0.564 & 0.958 & 0.351 & 0.775 & 0.929 \\
\hline IGT/CCT & & & & & & & -0.142 & -0.167 & 0.028 & -0.175 & 0.021 & 0.234 & -0.041 & -0.060 \\
\hline$p$ & & & & & & & 0.381 & 0.302 & 0.864 & 0.279 & 0.896 & 0.146 & 0.800 & 0.711 \\
\hline
\end{tabular}

Table 2. Correlation of each parameter in the KC and others groups: Pearson correlation coefficients. Underline values indicate $p<0.05$. KC keratoconus, $P K$ penetrating keratoplasty, Postop period postoperative period, $A C D$ anterior chamber depth, IHT inferior thinnest host cornea thickness, CCT central corneal thickness, IGT inferior thinnest graft thickness, IHT/CCT IHT ratio to CCT, IGT/CCT IGT ratio to CCT, Ks steepest corneal power, AveK average corneal power, CYL cylinder, Sph spherical, Reg regular astigmatism, Asym asymmetry, $\mathrm{HOI}$ higher order irregularity, $K_{\max }$ maximum corneal power.

postoperative period, sex, and graft size), postoperative period and graft size were selected. In other words, the KC could progress over time after PK and a smaller graft might lead to a more severe progression. The fact that the postoperative period was selected is reasonable given the progressive nature of KC. As for the reason graft size was selected, we speculate that a small graft might be inadequate for removing all the affected KC lesion. Thus, surgeons who perform PK in KC eyes should be careful to choose an adequately sized graft.

Whether it is the host cornea or the graft that develops $\mathrm{KC}$ progression has remained controversial. The finding that both IHT/CCT and IGT/CCT were smaller in the KC group than in the Others group supports the notion that the progression occurs in both the graft and host. This notion is also supported by our previous reports on histopathologic specimens of corneas with recurrent $\mathrm{KC}^{7,8}$. In the regression analysis that we performed to find out the factors contributing to the keratometric changes, IHT/CCT, a parameter reflecting host thinning, was selected in the analyses. In contrast, IGT/CCT, reflecting graft thinning, was not selected. This means that keratometric changes occurred mainly because of changes to the host cornea.

A limitation of the study is that we did not evaluate the lens status, e.g., phakic or pseudophakic, which could affect the ACD, a protrusion parameter. As the ACD correlated with the corneal steepening in both groups, however, the effect of lens status was likely minimal. Another limitation is that we did not show the data at the time of PK. However, there should not have been big differences in tomographic data between the groups just after PK. For example, KC eyes tend to have deeper ACD, but postoperative normalization of ACD by PK has been reported ${ }^{19,20}$. Also, inferior graft thinning, which was observed only in KC group, should not have existed at the time of PK. 
KC
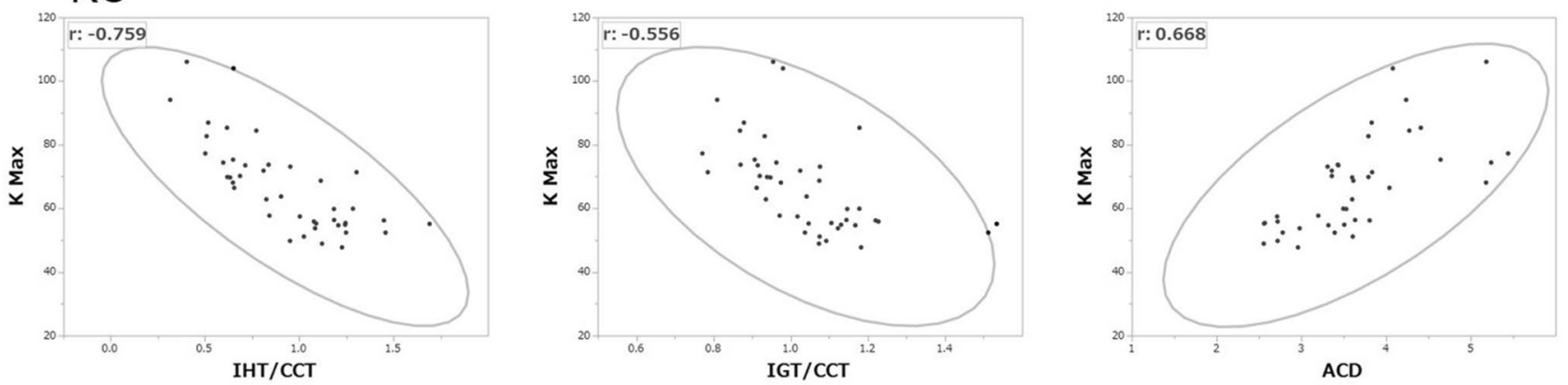

\section{Others}

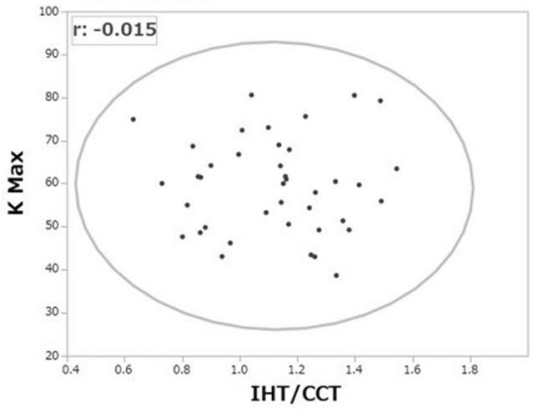

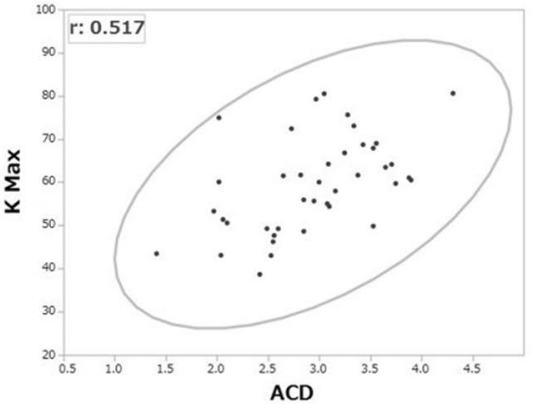

Figure 3. Scatterplots of $\mathrm{K}_{\max }$ and the corneal ectasia indicators (IHT/CCT, IGT/CCT, and ACD) in the KC (upper graphs) and Others (lower graphs) groups. $\mathrm{K}_{\max }$ was strongly correlated with host and graft thinning in the KC group but not in the Others group. Deeper ACD correlated with a larger $\mathrm{K}_{\max }$ in both groups. Density ellipses show $\mathrm{p}=0.990$.

\begin{tabular}{|c|c|c|c|c|c|c|}
\hline \multirow[b]{2}{*}{ Dependent } & \multicolumn{3}{|l|}{ KC } & \multicolumn{3}{|l|}{ Others } \\
\hline & Selected & Estimates & $p$ & Selected & Estimates & $p$ \\
\hline \multirow{2}{*}{ IHT/CCT } & Postoperative period & 0.012 & 0.021 & \multirow{2}{*}{ Age at PK } & \multirow{2}{*}{0.005} & \multirow{2}{*}{0.004} \\
\hline & Graft size & 0.286 & $<0.0001$ & & & \\
\hline \multirow{2}{*}{ IGT/CCT } & Postoperative period & 0.006 & 0.019 & \multirow{2}{*}{ Age at $\mathrm{PK}$} & \multirow{2}{*}{0.003} & \multirow{2}{*}{0.067} \\
\hline & Graft size & 0.155 & $<0.0001$ & & & \\
\hline \multirow{2}{*}{ ACD } & Postoperative period & -0.026 & 0.075 & Postoperative period & -0.017 & 0.044 \\
\hline & Graft size & -0.418 & 0.019 & Graft size & 0.310 & 0.036 \\
\hline
\end{tabular}

Table 3. Factors associated with corneal ectasia indicators in $\mathrm{KC}$ and others: multivariant regression analysis of IHT/CCT, IGT/CCT, and ACD as the dependent variables with the independent variables of age at PK, current age, postoperative period, sex, and graft size. KC keratoconus, IHT inferior thinnest host cornea thickness, CCT central corneal thickness, IGT inferior thinnest graft thickness, IHT/CCT IHT ratio to CCT, IGT/CCT IGT ratio to CCT, ACD Anterior chamber depth, PK Penetrating keratoplasty.

In conclusion, KC eyes long after PK tend to show inferior graft and host corneal thinning, and corneal protrusion, suggesting $\mathrm{KC}$ progression. Corneal power parameters such as the $\mathrm{K}_{\max }$ or $\mathrm{Ks}$ can be used to evaluate $\mathrm{KC}$ progression after PK, but astigmatic parameters such as CYL cannot. Although both host and graft showed $\mathrm{KC}$ changes, keratometric changes mainly resulted from host corneal thinning. When performing PK in KC patients, it is important to select an adequately sized graft.

\section{Methods}

The study was approved by the ethics committee of the University of Tokyo Hospital. Requirements of informed consent were waived for this retrospective chart review by the ethics committee of the University of Tokyo Hospital. Data analysis was performed on de-identified data, in accordance with the relevant guidelines and regulations.

Subjects in this study were recruited from among those who visited the University of Tokyo Hospital Cornea clinic between December 2015 and February 2016. Seventy-six patients who had maintained clear grafts for more 


\begin{tabular}{|c|c|c|c|c|c|c|}
\hline \multirow[b]{2}{*}{ Dependent } & \multicolumn{3}{|l|}{ KC } & \multicolumn{3}{|l|}{ Others } \\
\hline & Selected & Estimates & $p$ & Selected & Estimates & $p$ \\
\hline Ks & IHT/CCT & -20.61 & $<0.0001$ & $\mathrm{ACD}$ & 3.25 & 0.034 \\
\hline \multirow{2}{*}{$\operatorname{AvgK}$} & IHT/CCT & -11.98 & 0.001 & \multirow{2}{*}{$\mathrm{ACD}$} & \multirow{2}{*}{2.99} & \multirow{2}{*}{0.032} \\
\hline & ACD & 3.17 & 0.036 & & & \\
\hline CYL & IHT/CCT & -7.77 & 0.013 & N/A & - & - \\
\hline \multirow{2}{*}{ Fourier Sph } & IHT/CCT & -11.22 & 0.003 & \multirow{2}{*}{$\mathrm{ACD}$} & \multirow{2}{*}{3.36} & \multirow{2}{*}{0.015} \\
\hline & ACD & 3.38 & 0.026 & & & \\
\hline Fourier Reg & IHT/CCT & -4.39 & 0.005 & N/A & - & - \\
\hline Fourier Asym & IHT/CCT & -8.41 & $<0.0001$ & IGT/CCT & 3.54 & 0.122 \\
\hline Fourier HOI & IHT/CCT & -0.88 & 0.0003 & ACD & 0.54 & 0.026 \\
\hline \multirow{2}{*}{$\mathrm{K}_{\max }$} & IHT/CCT & -28.30 & $<0.0001$ & \multirow{2}{*}{$\mathrm{ACD}$} & \multirow{2}{*}{8.90} & \multirow{2}{*}{0.001} \\
\hline & $\mathrm{ACD}$ & 6.05 & 0.016 & & & \\
\hline
\end{tabular}

Table 4. Factors associated with corneal powers and astigmatism in the KC and Others groups: Multivariant regression analysis on AS-OCT parameters as dependent variables with the independent variables IHT/CCT, IGT/CCT and ACD. KC keratoconus, AS-OCT anterior segment optical coherence tomography, IHT inferior thinnest host cornea thickness, CCT central corneal thickness, IGT inferior thinnest graft thickness, IHT/CCT IHT ratio to CCT, IGT/CCT IGT ratio to CCT, $A C D$ anterior chamber depth, Ks steepest corneal power, AvgK average corneal power, CYL cylinder, Sph spherical, Reg regular astigmatism, Asym asymmetry, HOI higher order irregularity, $K_{\max }$ maximum corneal power.

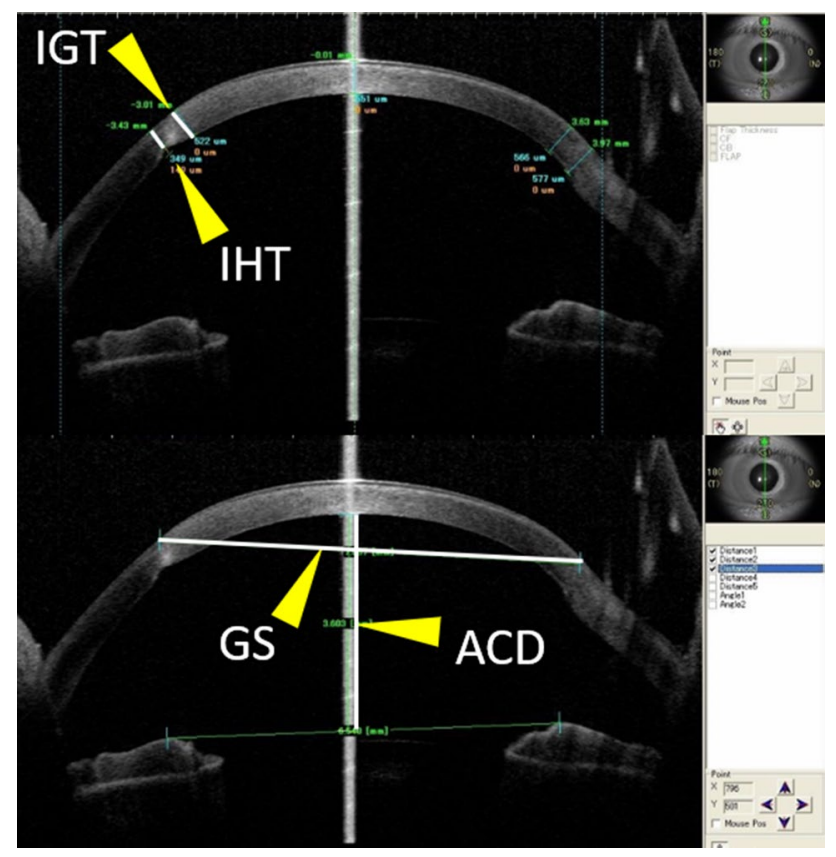

Figure 4. Scheme of each parameter measured by anterior segment optical coherence tomography. Vertical images were used for measurements. The thicknesses of the thinnest parts of the inferior graft (IGT) and host (IHT) cornea, graft size (GS), and anterior chamber depth (ACD) were measured.

than 10 years after PK and had undergone AS-OCT (CASIA, SS-1000/SS-2000, Tomey, Japan) were enrolled in this study. The patients were divided into 2 groups according to the primary indication for PK: KC or Others groups. Patient age at PK (age at PK), number of years after PK when AS-OCT was performed (postoperative period), current age and sex were recorded. Graft size, ACD, CCT, IHT, and IGT were measured from AS-OCT vertical images (Fig. 4). The ratios of IHT and IGT to CCT (IHT/CCT and IGT/CCT) were calculated as the inferior corneal thinning indicators. ACD was used to evaluate corneal protrusion. In the present article, we refer to IHT/CCT, IGT/CCT, and ACD as corneal ectasia indicators. Keratometric parameters, such as Ks, AvgK, CYL, Fourier analysis parameters (Sph, Reg, Asym and HOI), and $\mathrm{K}_{\max }$ were also obtained from AS-OCT. The data were compared between the 2 groups. Correlations were examined between each parameter except between the keratometric parameters in both groups. Multivariate regression analyses were performed in each group 
to determine the risk factors associated with corneal ectatic change and to determine the factors associated with keratometric changes. To determine corneal ectasia risk factors, 5 possible explanatory variables (age at $\mathrm{PK}$, current age, postoperative period, sex, and graft size) were evaluated with the objectives of corneal ectasia indicators (IHT/CCT, IGT/CCT, and ACD). To determine the factors associated with keratometric changes, the 3 explanatory variables of corneal ectasia indicators (IHT/CCT, IGT/CCT, and ACD) were examined with the objectives of the AS-OCT keratometric parameters (Ks, AvgK, CYL, Sph, Reg, Asym, and $\mathrm{K}_{\max }$ ).

Statistical analysis. Data comparison was performed by t-test, except the man to woman ratio, which was analyzed by Fisher's exact test. Pearson correlation coefficients were obtained for the correlation analysis. A parameter combination with minimum corrected AIC was selected by the stepwise method to determine the best model for each multivariate regression analysis. A $p<0.05$ was considered statistically significant. The statistical analyses were performed using JMP Pro 13 (SAS Institute, Cary, NC, USA).

Received: 15 April 2020; Accepted: 14 October 2020

Published online: 29 October 2020

\section{References}

1. Krachmer, J. H., Mannis, M. J. \& Holland, E. J. Cornea 3rd edn. (Mosby, Maryland Heights, 2011).

2. Krachmer, J. H., Feder, R. S. \& Belin, M. W. Keratoconus and related noninflammatory corneal thinning disorders. Surv. Ophthalmol. 28, 293-322. https://doi.org/10.1016/0039-6257(84)90094-8 (1984).

3. Godefrooij, D. A., de Wit, G. A., Uiterwaal, C. S., Imhof, S. M. \& Wisse, R. P. Age-specific incidence and prevalence of keratoconus: a nationwide registration study. Am. J. Ophthalmol. 175, 169-172. https://doi.org/10.1016/j.ajo.2016.12.015 (2017).

4. Thompson, R. W., Price, M. O., Bowers, P. J. \& Price, F. W. Long-term graft survival after penetrating keratoplasty. Ophthalmology 110, 1396-1402. https://doi.org/10.1016/S0161-6420(03)00463-9 (2003).

5. Inoue, K., Amano, S., Oshika, T., Sawa, M. \& Tsuru, T. A 10-year review of penetrating keratoplasty. Jpn. J. Ophthalmol. 44, 139-145 (2000).

6. Meyer, J. J., Gokul, A., Crawford, A. Z. \& McGhee, C. N. Penetrating keratoplasty for keratoconus with and without resolved corneal hydrops: long-term results. Am. J. Ophthalmol. 169, 282-289. https://doi.org/10.1016/j.ajo.2016.07.001 (2016).

7. Fujita, A., Yoshida, J., Toyono, T., Usui, T. \& Miyai, T. Severity Assessment of acute hydrops due to recurrent keratoconus after penetrating keratoplasty using anterior segment optical coherence tomography. Curr. Eye Res. 44, 1189-1194. https://doi. org/10.1080/02713683.2019.1629597 (2019).

8. Yoshida, J. et al. Characteristics and risk factors of recurrent keratoconus over the long term after penetrating keratoplasty. Graefes Arch. Clin. Exp. Ophthalmol. 256, 2377-2383. https://doi.org/10.1007/s00417-018-4131-5 (2018).

9. Niziol, L. M., Musch, D. C., Gillespie, B. W., Marcotte, L. M. \& Sugar, A. Long-term outcomes in patients who received a corneal graft for keratoconus between 1980 and 1986. Am. J. Ophthalmol. 155, 213-219.e213. https://doi.org/10.1016/j.ajo.2012.08.001 (2013).

10. Pramanik, S., Musch, D. C., Sutphin, J. E. \& Farjo, A. A. Extended long-term outcomes of penetrating keratoplasty for keratoconus. Ophthalmology 113, 1633-1638. https://doi.org/10.1016/j.ophtha.2006.02.058 (2006).

11. Wollensak, G., Spoerl, E. \& Seiler, T. Riboflavin/ultraviolet-a-induced collagen crosslinking for the treatment of keratoconus. Am. J. Ophthalmol. 135, 620-627. https://doi.org/10.1016/s0002-9394(02)02220-1 (2003).

12. Huang, J. et al. Two-year topographic and densitometric outcomes of accelerated $(45 \mathrm{~mW} / \mathrm{cm}(2))$ transepithelial corneal crosslinking for keratoconus: a case-control study. BMC Ophthalmol. 18, 337. https://doi.org/10.1186/s12886-018-0999-4 (2018).

13. Meiri, Z. et al. Efficacy of corneal collagen cross-linking for the treatment of keratoconus: a systematic review and meta-analysis. Cornea 35, 417-428. https://doi.org/10.1097/ico.0000000000000723 (2016).

14. Filippello, M., Stagni, E. \& O’Brart, D. Transepithelial corneal collagen crosslinking: bilateral study. J. Cataract. Refract. Surg. 38, 283-291. https://doi.org/10.1016/j.jcrs.2011.08.030 (2012).

15. Mazzotta, C. et al. Corneal collagen cross-linking with riboflavin and ultraviolet a light for pediatric keratoconus: ten-year results. Cornea 37, 560-566. https://doi.org/10.1097/ico.0000000000001505 (2018).

16. Li, W. \& Wang, B. Efficacy and safety of transepithelial corneal collagen crosslinking surgery versus standard corneal collagen crosslinking surgery for keratoconus: a meta-analysis of randomized controlled trials. BMC Ophthalmol. 17, 262. https://doi. org/10.1186/s12886-017-0657-2 (2017).

17. Shajari, M. et al. Comparison of standard and accelerated corneal cross-linking for the treatment of keratoconus: a meta-analysis. Acta Ophthalmol. 97, e22-e35. https://doi.org/10.1111/aos.13814 (2019).

18. Pedrotti, E. et al. Topographic and biomechanical changes after application of corneal cross-linking in recurrent keratoconus. Int. J. Environ. Res. Public Health https://doi.org/10.3390/ijerph16203872 (2019).

19. Kim, K. H., Choi, S. H., Ahn, K., Chung, E. S. \& Chung, T. Y. Comparison of refractive changes after deep anterior lamellar keratoplasty and penetrating keratoplasty for keratoconus. Jpn. J. Ophthalmol. 55, 93-97. https://doi.org/10.1007/s10384-010-0914-x (2011).

20. Ort, A. et al. Evaluation of the cornea and anterior chamber morphologic changes after penetrating keratoplasty in patients with keratoconus. Eye Contact Lens 43, 236-239. https://doi.org/10.1097/icl.0000000000000260 (2017).

\section{Acknowledgements}

This work was supported by JSPS KAKENHI (Grant No. JP18K16917). The manuscript was carefully edited by a native-English-speaking professional editor from SciTechEdit international (https://www.scitechedit.com/).

\section{Author contributions}

J.Y. conceptualized, designed, analyzed data, and wrote and edited the main manuscript text. T.T., R.S., T.M, and T.U. reviewed and edited the manuscript. All authors contributed to the review and approval of the manuscript.

\section{Competing interests}

The authors declare no competing interests.

\section{Additional information}

Correspondence and requests for materials should be addressed to J.Y. 
Reprints and permissions information is available at www.nature.com/reprints.

Publisher's note Springer Nature remains neutral with regard to jurisdictional claims in published maps and institutional affiliations.

(c) (i) Open Access This article is licensed under a Creative Commons Attribution 4.0 International License, which permits use, sharing, adaptation, distribution and reproduction in any medium or format, as long as you give appropriate credit to the original author(s) and the source, provide a link to the Creative Commons licence, and indicate if changes were made. The images or other third party material in this article are included in the article's Creative Commons licence, unless indicated otherwise in a credit line to the material. If material is not included in the article's Creative Commons licence and your intended use is not permitted by statutory regulation or exceeds the permitted use, you will need to obtain permission directly from the copyright holder. To view a copy of this licence, visit http://creativecommons.org/licenses/by/4.0/.

(C) The Author(s) 2020 